\title{
Study of Behavioral Deficits Elicited by Exposure to Lipopolysacaride /Chronic Mild Stress in Male Wistar Rats \\ Mai Ahmed Ebeid ${ }^{1}$, Sawsan Aboul-Fotouh ${ }^{1,2}$, Ahmed Mohyeldin Abdel-tawab ${ }^{1,2}$, Omnyah Aly El-Kharashi ${ }^{1}$, Hadwa Ali Abd Al-khalek ${ }^{3}$ \\ ${ }^{1}$ Department of Pharmacology, ${ }^{2}$ Clinical Pharmacology Unit, ${ }^{3}$ Department of Histology Faculty of Medicine, Ain Shams University, Cairo, Egypt
}

Corresponding Author: Mai Ahmed Ebeid, E-mail: Mai_ebeid@med.asu.edu.eg, Tel: +20 1200520090

\begin{abstract}
Background: depression may be considered "the disease of the modern era". With a high prevalence worldwide causing significant morbidity and mortality and constituting a heavy burden on social and economic welfare. Despite decades-long research the exact pathogenesis of depression is still not fully understood. Aims: to investigate the behvioral deficits elicited by exposure to the combined model of lipopolysaccharide (LPS) then chronic mild stress (CMS) in male Wistar rats and to investigate the putative role of inflammatory cytokine production induced lipopolysaccharide exposure together with chronic stress in the pathogenesis of depressive like behaviour. Materials and Methods: Sixty five Male Wistar rats were divided into two groups; Control group $(\mathrm{n}=29)$ : naïve rats left undisturbed, not exposed to stress and LPS/CMS exposed group $(\mathrm{n}=27)$. Results: exposure to LPS/CMS model induced a depressive-like behavior manifested by a decline in body weight gain, alongside an increase in immobility time in FST and a decrease in time of active interaction in SIT. Conclusion: this work highlights the depressive like behaviour induced by exposure to the combined model of lipopolysaccharide and chronic mild stress. This work also underscores the putative role of lipopolysaccharide exposure in modulating the stress-induced neuroinflammation which is thought to be crucial, not only for the pathogenesis of depression, but also for a wide array of neurological diseases.
\end{abstract}

Key words: depression; chronic mild stress; lipopolysaccharide; forced swimming test; open field test; social interaction test.

\section{INTRODUCTION}

Depression is a common mental disorder that presents with depressed mood, loss of interest or pleasure, feelings of guilt and poor concentration. According to $\mathrm{WHO}$ it is the 4th leading contributor to the global burden of disease in 2000. By the year 2020, depression is projected to reach 2 nd place calculated for all ages, both sexes. This makes depression a major concern to the personal and economic welfare ${ }^{(1)}$. However, despite extensive biological research, the pathophysiology of depression is still elusive and treatments that target the causal factors of depression are not available ${ }^{(2)}$.

Over the last two decades, there has been growing evidence that inflammatory processes and neural-immune interactions are involved in the pathogenesis of major depression and may underlie some of serotonergic and adrenocortical correlates. This hypothesis was termed the monocyte-Tlymphocyte or cytokine hypothesis of depression ${ }^{(3)}$.

It is well-known that LPS (either peripheral or central), brain neuroinflammation and the increased production of pro-inflammatory cytokines, such as IL-1 $\beta$, IL-6 and TNF- $\alpha$, may induce specific symptoms, labeled as the sickness behavior syndrome ${ }^{(4)}$. Symptoms of sickness behavior, such as anorexia, reduction of locomotor activity and exploration, anhedonia and cognitive disturbances, bear a strong similarity with those of depression ${ }^{(5)}$.

Systematic validation of the endotoxin- or cytokine-induced anhedonia as an animal model of depression is not consistent. The striking similarities between symptoms of MDD in humans and the behavioral and physiological alterations induced by endotoxin or cytokine exposure in rodents offer a convincing 'face validity' for this model ${ }^{(6)}$. So that, we proposed in the present work that combined model of LPS then CMS could be a plausible model of depression that reflect some depressive disorders in human.

\section{MATERIALS AND METHODS}

\section{Animals}

Sixty five Male Wistar rats weighed from 200-300 g were purchased from the National research center, El-Giza, Egypt. Rats were allowed at least 1 week to acclimatize to the lab conditions. Rats' chow was purchased from Meladco for Animal Food, El-Obour, Egypt. Pellets and tap water were provided ad libitum unless otherwise recommended by the study protocol. The temperature was maintained at $24^{\circ} \mathrm{C}$. A $12 / 12 \mathrm{~h}$ light/dark cycle was maintained with lights on at 5 am. Animals were housed, each in one cage in the above conditions, unless otherwise recommended by the study protocol. All procedures were done 
according to expected ethical conduct in animal research.

\section{Treatments and experimental groups}

Rats were divided into two groups; Control group $(n=29)$ : naïve rats left undisturbed, not exposed to stress and LPS/CMS exposed group $(\mathrm{n}=27)$ : LPS injected followed by CMS exposure.

\section{Experimental models}

\subsection{LPS injection: according to Elgarf et al. ${ }^{(7)}$}

All animal groups of combined LPS then CMS model were exposed to LPS in a dose of $50 \mu \mathrm{g} /$ $\mathrm{kg}$ for six i.p. injections every other day over 2 weeks.

\subsection{Chronic Mild Stress (CMS): according to Willner et al. ${ }^{(8)}$}

The chronic mild stress was applied in a semi-random sequence to be unpredictable with the assumption that this will lead to a reduction in their sensitivity to reward (anhedonia). The stressors were food or water deprivation; cage tilting; reversed light cycle; pairing; stroboscopic light (60 flashes/min); intermittent white noise $(85 \mathrm{~dB})$; cold temperature $\left(10^{\circ} \mathrm{C}\right)$; restricted access to food (3 pellets); empty water bottles, foreign body in the cage, and soiling of cage with $50-100 \mathrm{ml}$ water. Pairing was done with different cage mate every time. Control animals were kept undisturbed and in separate cages.

\section{Behavioral Tests}

The body weight of rats was measured at the beginning of the experiment (baseline weight) then weekly thereafter. Behavioral tests were done during the last 10 days of the experiment. The sequence of applying behavioral tests was sucrose preference test (SPT), open field test (OFT), social interaction test (SIT), then forced swimming test (FST) preceded one day with forced swimming test training.

4.1. Sucrose preference test (SPT): according to Chiba et al. ${ }^{(9)}$

During the $5^{\text {th }}$ week of the study, Animals were acclimatized for one week at the site specifically prepared to host the experiment. Then, rats were trained for one week to consume a palatable weak (1\%) sucrose solution. Training consisted of exposure to $1 \%$ sucrose solution in one bottle, together with tap water in another bottle. The position of bottles was exchanged every day to avoid habituation to the site of sucrose. The test was done on the $6^{\text {th }}$ week from Tuesday 8 am to Wednesday 8 a.m. Rats were given, for $24 \mathrm{~h}$, a free choice between the two $200 \mathrm{ml}$ bottles (one contained tap water and the other contained 1\% sucrose solution) and consumption of water and sucrose solution was calculated by subtracting the weight of the bottles after $24 \mathrm{~h}$. Sucrose preference was calculated as a percentage consumed sucrose solution of the total fluid intake.

The sucrose preference will be calculated according to the following formula: sucrose preference $(\%)=($ sucrose intake/total fluid intake) X100.

4.2. Open Field Test (OFT): according to Tonissaar et al. ${ }^{(10)}$

Open field test was used to detect general locomotor activity in rats. On Tuesday of $5^{\text {th }}$ week, the rats were allowed to acclimatize to the test room 1 $\mathrm{hr}$ before conducting the experiment. Each rat was placed individually in the centre of Quadrangular arena $(60 \times 60 \mathrm{~cm})$ with $45 \mathrm{~cm}$ height walls; the arena is divided into 16 equal squares illuminated by white light. The test duration was conducted for 5 minutes to each rat. Behavior was videotaped and the number of crossed squares (with at least three paws), time spent in central zone, frequency of rearing (standing upright on the hind paws), and grooming including face cleaning, paw licking, fur licking, head scraping and rubbing will be counted manually. The arena was cleaned by $10 \%$ alcohol after each rat.

\subsection{Social Interaction Test (SIT): according to Tonissaar et al. ${ }^{(11)}$}

In SIT two unfamiliar weight-matched rats from the same group were placed in opposite corners of a well-illuminated chamber $(30 \times 30 \times 60$ $\mathrm{cm}$ ) with the floor covered with wood shavings for $10 \mathrm{~min}$. The behavior was videotaped and total time spent in active social behavior (allogrooming, sniffing the partner, crawling under and over, following) was calculated, for each rat, separately.

\subsection{Forced Swimming Test (FST): according to Porsolt et al. ${ }^{(12)}$}

The FST involves immersion of animals in a Cylindrical Plexiglas tank (diameter $22 \mathrm{~cm}$, height $50 \mathrm{~cm})$ filled with $(35 \mathrm{~cm})$ ambient water maintained at $25^{\circ} \mathrm{C}$. On Wednesday of the $5^{\text {th }}$ week, rats were trained to swim for 10-15 min. Water was changed after testing of each animal. One day later rats were re-exposed to the forced swimming for 5 minutes. Behavior was videotaped and immobility time was measured with a stopwatch. Immobility time is the time during which the animal floats on 
the surface with its front paws together and makes only those movements which were necessary to keep it afloat. According to an original version of the FST, the test was performed on each rat only once; on Thursday $5^{\text {th }}$ week of the study.

\section{Statistical Analysis}

Prism 5.01 (GraphPad Software, La Jolla, CA, USA) was used for all the graphical presentations and statistical analyses. Mannwhitney test for non-parametric data was used to compare the two groups. For body weight, repeated-measure analysis of variance (ANOVA) with Bonferroni's post-hoc test was used. The statistical significance level was set at $p<0.05$.

\section{Ethical and approval statements}

All animal procedures followed the international guideline of proper experimental animal handling. The present study was approved by the Research Ethics Committee of Faculty of Medicine, Ain Shams University (FMASU-REC). FMASUREC operates under Federal Wide Assurance.

\section{RESULTS}

\section{Effect of exposure to LPS/CMS model on body weight gain in Wistar rats}

As shown in figure (1), repeated-measure ANOVA revealed a significant main effect of time $\left(F_{(5,156)}=47.13, P<0.0001\right)$ and stress $\left(F_{(1,156)}=112.94\right.$, $\mathrm{P}<0.0001)$. Rats exposed to LPS/CMS model showed a significant decrease in body weight gain as compared to their respective controls at the end of the 2nd, 3rd, 4th, 5th and 6th weeks $(\mathrm{P}<0.001)$.

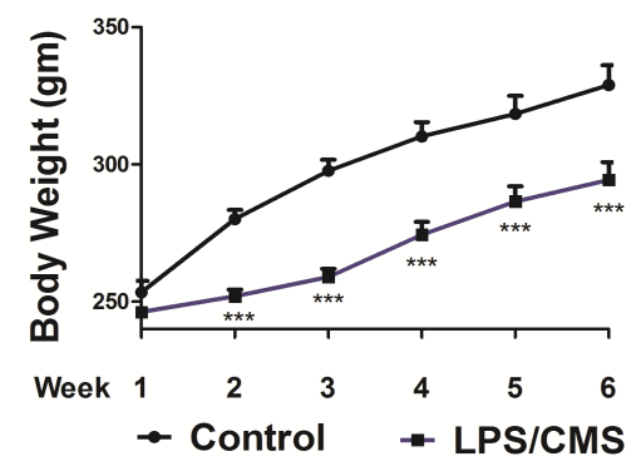

Figure (1): Effects of exposure to LPS/CMS model on body weight in Wistar rats. Data are mean \pm SEM of 13 15 animals per group. ${ }^{* * *} \mathrm{P}<0.001$ vs. Control group by repeated-measure ANOVA with Bonferroni's post-hoc test.

\section{Effect of exposure to LPS/CMS model on sucrose preference test in Wistar rats}

As shown in figure (2), Wistar rats exposed to LPS/CMS model exhibited a significant decrease $(\mathrm{p}<0.001)$ in SPT compared to control rats.

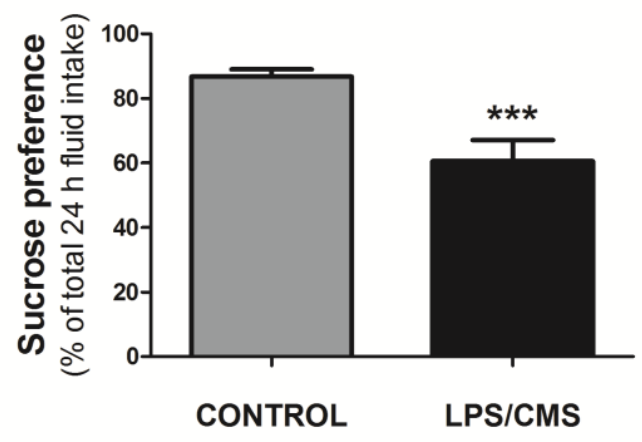

Figure (2): Effects of exposure to LPS / CMS model on sucrose preference test in Wistar rats. Data are mean \pm SEM of $15-16$ animals per group. ${ }^{* * *} \mathrm{P}<0.001$ vs. Control group; by Mann-Whitney Test.

\section{Effect of exposure to LPS/CMS model on open field test in Wistar rats}

As shown in Figure (3), only, latency to leave central zone was significantly reduced in rats exposed to LPS/CMS model $(\mathrm{p}<0.05)$ as compared to the control rats. Exposure to LPS/CMS model did not induce a significant difference in total number of crossed squares, time spent in central zone or frequency of entering central zone as compared to the control rats.

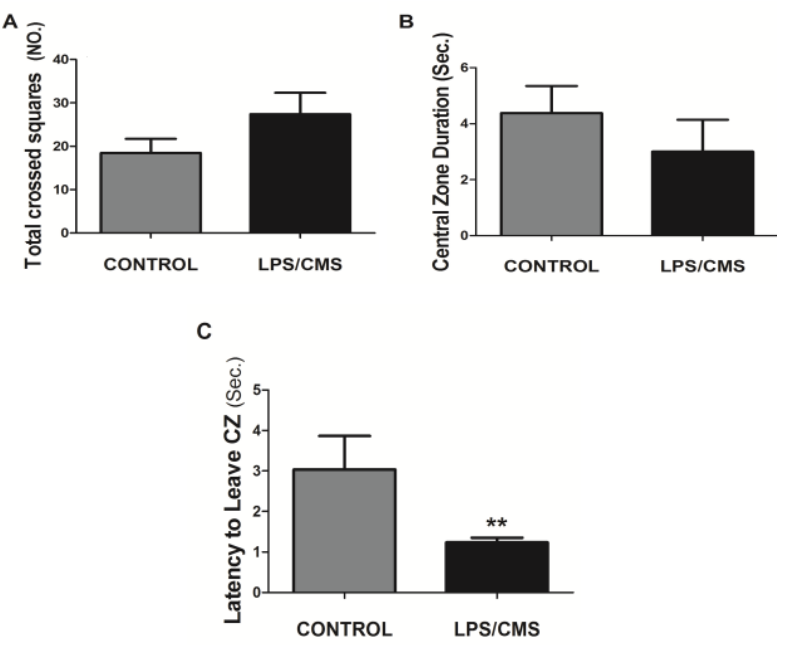

Figure (3): Effects of exposure to LPS / CMS model on open field test (OFT) in Wistar rats. Data are mean \pm SEM of 15-21 animals per group. ${ }^{* *} \mathrm{P}<0.01$ vs. Control group by Mann-Whitney test. 


\section{Effect of exposure to LPS/CMS model on social interaction test in Wistar rats}

As shown in figure (4), Wistar rats exposed to LPS/CMS model exhibited a significant decline in time of active interaction with its partner as compared to their respective controls $(\mathrm{p}<0.05)$.

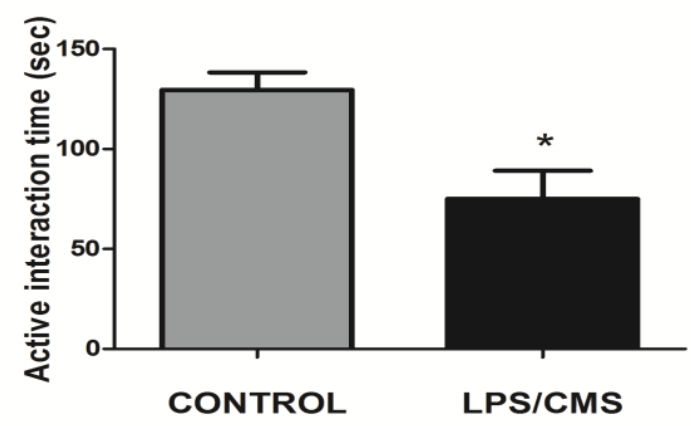

Figure (4): Effects of exposure to LPS / CMS model on social interaction test (OFT) in Wistar rats. Data are mean \pm SEM of 10 animals per group. ${ }^{*} \mathrm{P}<0.01$ vs. Control group by Mann-Whitney test.

\section{Effect of exposure to LPS/CMS model on forced swimming test in Wistar rats}

As shown in figure (5), Wistar rats exposed to LPS/CMS model exhibited a significant increase in immobility time $(\mathrm{p}<0.001)$ alongside an increase in swimming time $(\mathrm{p}<0.05)$ and a decline in struggling time $(\mathrm{p}<0.001)$.

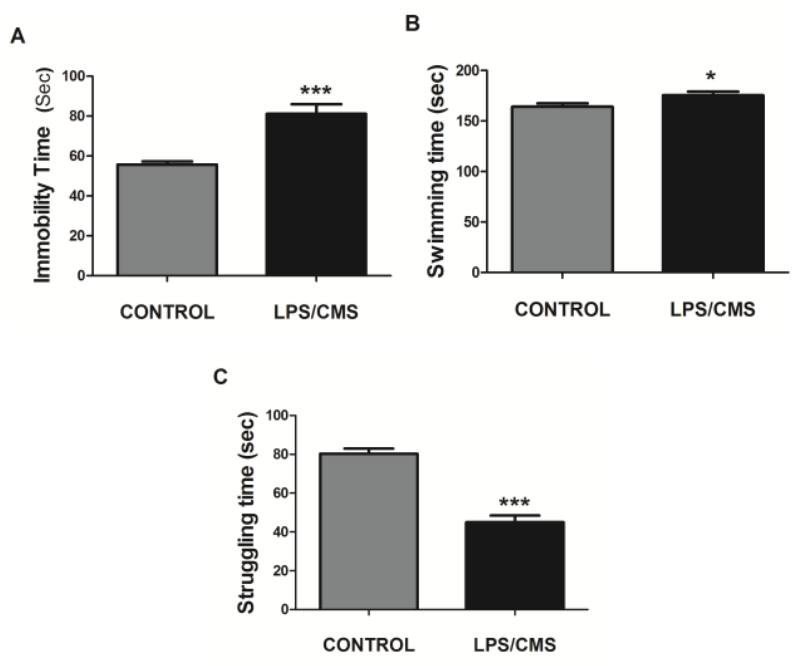

Figure (5): Effects of exposure to LPS / CMS model on forced swimming test (FST) in Wistar rats. Data are mean \pm SEM of 15-21 animals per group. ${ }^{*} \mathrm{P}<0.05$, ${ }^{* * * *} \mathrm{P}<0.001$ vs. Control group by Mann-Whitney test.

\section{DISCUSSION}

Depression may be considered "the disease of the modern era" ${ }^{(13)}$.With a high prevalence worldwide (14), significant morbidity and mortality, depression constitutes a heavy burden on social and economic welfare. Despite decades-long research the exact pathogenesis of depression is still not fully understood.

Over years, many theories have been proposed to explain the pathophysiology of depression with an increasing body of evidence linking activation of immune-inflammatory responses to the pathogenesis of depression ${ }^{(15)}$. In this context, microglia are effectively involved in regulating the immune environment of the central nervous system with production of pro-inflammatory cytokines which are eventually involved in the pathogenesis of multiple disorders involving major depression ${ }^{(16)}$.

This study was designed to investigate the behvioral deficits elicited by exposure to the combined model of LPS then CMS in male Wistar rats. This is to investigate the putative role of inflammatory cytokine production induced lipopolysaccharide exposure together with chronic stress in the pathogenesis of depressive like behaviour.

In the present work, exposure to LPS then CMS model was able to induce a depressive like behaviour manifested by a decrease in body weight, decreased sucrose preference, increased immobility time alongside decreased struggling times on FST when compared to their respective controls.

The prolonged immobility time and reduced struggling time in the forced swim test are used to infer the development of depressive like behaviour (13). Increased immobility could be described as "a corollary" of stress-induced anhedonia ${ }^{(17)}$. Moreover, the increased entry to the central zone in OFT represents an index of anxiety associated with depression ${ }^{(18)}$. Hyperactivity of HPA axis is well documented event in depression and the dysregulation of the HPA axis has been regarded as a neuro-endocrine hallmark of chronic stress ${ }^{(19)}$, the significantly reduced body weight gain and increased serum corticosterone level in rats exposed to LPS/CMS indicates the absence of an adequate adaptive response to stress ${ }^{(20) \text {. }}$

For many years, the chronic mild stress model of depression was the most widely used model of depression, extensively used by researchers to investigate many aspects of depression. Indeed, reduced sucrose preference, increased immobility 
time and reduced struggling times in FST manifested in our CMS-exposed rats are centered on the core symptom of depression, anhedonia ${ }^{(21)}$.

The systemic increase in LPS is associated with systemic inflammation, activation of microglia and enhanced neuroinflammation ${ }^{(22)}$. LPS activates TLR-4 with subsequent induction of the expression of proinflammatory cytokines; IL-1, IL-6 and TNF- $\alpha$. The main sources of these pro-inflammatory cytokines within the brain are microglia and perivascular and meningeal macrophages ${ }^{(23)}$. Notably, disturbances in brain neurotransmitter levels is well documented with repeated LPS challenges, studies reported significant reduction in dopamine and serotonin levels in the prefrontal cortex and hippocampus of LPS-treated rats alongside an increased concentration of glutamate ${ }^{(24)}$.

In the same context, Stress is critical for LPS-associated activation of microglia and subsequent neuroinflammation, LPS treatment failed to activate microglia in hippocampus of nonstressed rats in comparison to a dramatic effect in activating microglia and astrogliosis in the stressexposed group ${ }^{(25)}$.

On the other side, a study ${ }^{(26)}$ reported that the combination of LPS challenge with CMS paradigm modulated the stress-induced behavioral changes with exacerbation of depressive and inhibition of aggressive behaviors.

The relatively less depressive state in the FST noticed in LPS/CMS exposed rats may infer the development of endotoxin tolerance in response to LPS repeated challenge. Endotoxin tolerance refers to the reduced responsiveness to endotoxin challenge after a primary bacterial insult with subsequent suppression of pro-inflammatory cytokine production (27). As an adaptive mechanism of the innate immune system, endotoxin tolerance protects the body against severe endotoxin mediated damage as septic shock (28). Indeed, many studies indicated the long-term desensitization of the HPA axis after a single LPS challenge, underscoring the development of adaptive response to stress ${ }^{(29)}$.

Elgarf and her colleagues ${ }^{(7)}$ reported that combining LPS repeated challenges to CMS had additive effect with exaggerated behavioral and neuro-inflammatory disturbances associated with chronic stress. This controversy suggests that LPS induced behavioral changes are dependent on the timing, frequency of exposure and dose of LPS alongside the duration and type of stress modality.

\section{CONCLUSION}

This work highlights the depressive like behaviour induced by exposure to the combined model of lipopolysaccharide and chronic mild stress. This work also underscores the putative role of lipopolysaccharide exposure in modulating the stressinduced neuroinflammation which is thought to be crucial, not only for the pathogenesis of depression, but also for a wide array of neurological diseases.

\section{CONFLICT OF INTEREST}

There were no conflicts of interest.

\section{REFERENCES}

1. Sobocki P, Jonsson B, Angst J, Rehnberg C (2006): Cost of depression in Europe. J. Ment. Health Policy Econ., 9(2):87-98

2. Maes M, Yirmyia R, Noraberg J, Brene S, Hibbeln J, Perini G, Kubera M, Bob P, Lerer B, Maj $M$ (2009). The inflammatory \& neurodegenerative (I\&ND) hypothesis of depression. leads for future research and new drug developments in depression. Metab. Brain Dis., 24:27-53

3. Schiepers OJG, Wichers MC, Maes M (2005): Cytokines and major depression. Progress in Neuro-Psychopharmacology \& Biological Psychiatry, 29(2): 201-217.

4. Qin L, Wu X, Block ML, Liu Y, Breese GR, Hong JS, Knapp DJ, and Crews FT (2007): Systemic LPS causes chronic neuroinflammation and progressive neurodegeneration. Glia, 55(5):453-462.

5. Yirmiya R (1997): Behavioral and psychological effects of immune activation: implications for 'depression due to a general medical condition'. Curr. Opin. Psychiatr., 10:470-476

6. De La Garza $R$ (2005): Endotoxin- or proinflammatory cytokine induced sickness behavior as an animal model of depression: focus on anhedonia. Neurosci. Biobehav. Rev., 29:761-770

7. Elgarf AS, Aboul-Fotouh S, Abd-Alkhalek HA, El Tabbal M, Hassan AN, Kassim SK, Hammouda GA, Farrag KA, Abdel-tawab AM (2014): Lipopolysaccharide repeated challenge followed by chronic mild stress protocol introduces a combined model of depression in rats: reversibility by imipramine and pentoxifylline. Pharmacol Biochem Behav., 126:152-62. 
8. Willner P, Muscat $R$ and Papp $M$ (1992). Chronic mild stress-induced anhedonia: A realistic animal model of depression. Neurosci Biobehav Rev., 16(4):525-534

9. Chiba S, Numakawa T, Ninomiya $\mathrm{M}$ et al. (2012): Chronic restraint stress causes anxietyand depression-like behaviors, downregulates glucocorticoid receptor expression, and attenuates glutamate release induced by brain derived neurotrophic factor in the prefrontal cortex. Prog Neuropsychopharmacol Biol Psychiatry, 39(1):112-19.

10. Tonisaar M, Mällo T, Eller M et al. (2008): Rat behaviour after chronic variable stress and partial lesioning of 5-HT-ergic neurotransmission: Effects of citalopram. Prog NeuroPsychopharmacol Biol Psychiatry, 32: 164-77.

11. Tonissaar M, Philips M, Eller $M$ et al. (2004): Sociability trait and serotonin metabolism in the rat social intraction test. Neurosci Lett., 367: 309 - 12.

12. Porsolt RD, Le Pichon M, Jalfre M (1977): Depression: a new animal model sensitive to antidepressant treatments. Nature., 266(5604):730-2.

13. Aboul-Fotouh S, Habib M, Asaad T, Kassim SK, Ghanem MH (2018): Behavioral effects of toll-like receptor-4 antagonist 'eritoran' in an experimental model of depression: role of prefrontal and hippocampal neurogenesis and $\gamma$ aminobutyric acid/glutamate balance. Behav Pharmacol., 29(5):413-425.

14. World Health Organization (2012): Depression: A Global Public Health Concern. Developed by Marcus M, Yasamy MT, van Ommeren M, Chisholm D and Saxena S; WHO Department of Mental Health and Substance Abuse. https://www.goinginternational.eu/news/115/

15. Gárate I, García-Bueno $B$, Madrigal $J$, Bravo L, Berrocoso E, Caso JR, Micó JA, Leza JC (2011): Origin and consequences of brain Toll-like receptor 4 pathway stimulation in an experimental model of depression. Journal of Neuroinflammation, 8:151-165.

16. Ransohoff RM, Brown MA (2012): Innate immunity in the central nervous system. J Clin Invest., 122(4):1164-71.
17. Willner P (2005): Chronic mild stress (CMS) revisited: consistency and behaviouralneurobiological concordance in the effects of CMS. Neuropsychobiology, 52(2):90-110.

18. Aboul-Fotouh S (2013): Coenzyme Q10 displays antidepressant-like activity with reduction of hippocampal oxidative/nitrosative DNA damage in chronically stressed rats. Pharmacol Biochem Behav., 104:105-12.

19. Habib M, Shaker S, El-Gayar N, AboulFotouh S (2015): The effects of antidepressants "fluoxetine and imipramine" on vascular abnormalities and Toll like receptor-4 expression in diabetic and non-diabetic rats exposed to chronic stress. PLoS One, 10(3):e0120559.

20. Aboul-Fotouh S (2015): Behavioral effects of nicotinic antagonist mecamylamine in a rat model of depression: prefrontal cortex level of BDNF protein and monoaminergic neurotransmitters. Psychopharmacology (Berl), 232(6):1095-105.

21. Willner $P$ (2016) :The chronic mild stress (CMS) model of depression: History, evaluation and usage. Neurobiol Stress, 24;6:78-93.

22. O'Connor JC, Lawson MA, André C, Moreau M, Lestage J, Castanon N, Kelley KW, Dantzer R (2009): Lipopolysaccharideinduced depressive-like behavior is mediated by indoleamine 2,3-dioxygenase activation in mice. Mol Psychiatry, 14(5):511-22.

23. Dantzer R (2001): Cytokine-induced sickness behavior: mechanisms and implications. Ann N Y Acad Sci., 933:222-34.

24. Dang R, Zhou $X$, Tang M, Xu P, Gong $X$, Liu Y, Jiao H, Jiang $P$ (2018): Fish oil supplementation attenuates neuroinflammation and alleviates depressive-like behavior in rats submitted to repeated lipopolysaccharide. Eur J Nutr., 57(3):893-906.

25. Espinosa-Oliva AM, de Pablos RM, Villarán RF, Argüelles S, Venero JL, Machado A, Cano J (2011): Stress is critical for LPS-induced activation of microglia and damage in the rat hippocampus. Neurobiol Aging, 32(1):85-102. 
26. Couch Y, Trofimov A, Markova N, Nikolenko V, Steinbusch HW, Chekhonin V, Schroeter C, Lesch KP, Anthony DC, Strekalova T (2016): Low-dose lipopolysaccharide (LPS) inhibits aggressive and augments depressive behaviours in a chronic mild stress model in mice. J Neuroinflammation, 16;13(1):108.

27. Yang Y, Sun H, Mo X, Liu Y, Jia H, Li X, Zhang G, Li Q(2012): Prediction of novel genes associated with negative regulators of toll-like receptors-induced inflammation based on endotoxin tolerance. Inflammation, 35(6):188999.
28. Gantner BN, Singh H (2007): Immunology: Short-term memory. Nature, 447(7147):916-7.

29. Liu S, Zhu X, Liu Y, Wang C, Wang S, Tang X, Ni X (2011): Endotoxin tolerance of adrenal gland: attenuation of corticosterone production in response to lipopolysaccharide and adrenocorticotropic hormone. Crit Care Med., 39(3):518-26. 